\title{
Diagnostic Ability of Convex-Arrayed Endoscopic Ultrasonography for Major Vascular Invasion in Pancreatic Cancer
}

\author{
Yuki Fujii ${ }^{1}$, Kazuyuki Matsumoto ${ }^{1}$, Hironari Kato ${ }^{1}$, Yosuke Saragai', Saimon Takada', Sho Mizukawa ${ }^{1}$, Shinichiro Muro ${ }^{1}$, \\ Daisuke Uchida', Takeshi Tomoda', Shigeru Horiguchi ${ }^{1}$, Noriyuki Tanaka ${ }^{2}$ and Hiroyuki Okada ${ }^{1}$ \\ Department of ${ }^{1}$ Gastroenterology and Hepatology, ${ }^{2}$ Pathology, Okayama University Graduate School of Medicine, Dentistry and \\ Pharmaceutical Science, Okayama, Japan
}

Background/Aims: This study aimed to examine the diagnostic ability of endoscopic ultrasonography (EUS) for major vascular invasion in pancreatic cancer and to evaluate the relationship between EUS findings and pathological distance.

Methods: In total, 57 consecutive patients who underwent EUS for pancreatic cancer before surgery were retrospectively reviewed. EUS image findings were divided into four types according to the relationship between the tumor and major vessel (types 1 and 2: invasion, types 3 and 4: non-invasion). We also compared the EUS findings and pathologically measured distances between the tumors and evaluated vessels.

Results: The sensitivity, specificity, and accuracy of EUS diagnosis for vascular invasion were $89 \%$, 92\%, and $91 \%$, respectively, in the veins and $83 \%, 94 \%$, and 93\%, respectively, in the arteries. The pathologically evaluated distances of cases with type 2 EUS findings were significantly shorter than those of cases with type 3 EUS findings in both the major veins (median [interquartile range], 96 [0-742] $\mu \mathrm{m}$ vs. $2,833[1,076-5,694] \mu \mathrm{m}, p=0.012)$ and arteries $(623[0-854] \mu \mathrm{m}$ vs. $3,097[1,396-6,000] \mu \mathrm{m}, p=0.0061)$. All cases with a distance of $\geq 1,000 \mu \mathrm{m}$ between the tumors and main vessels were correctly diagnosed.

Conclusions: Tumors at a distance $\geq 1,000 \mu \mathrm{m}$ from the main vessels were correctly diagnosed by EUS. Clin Endosc 2019;52:479-485

Key Words: Endoscopic ultrasonography; Pancreatic cancer; Pathological distance; Staging; Vascular invasion

\section{INTRODUCTION}

The incidence of pancreatic cancer has gradually increased in the 20th and early 21 st century. ${ }^{1,2}$ In fact, pancreatic cancer is the sixth most common cancer and one of the leading causes of cancer-related mortality. ${ }^{1-3}$ Pancreatic cancer is associated

Received: September 23, 2018 Revised: January 3, 2019

Accepted: January 3, 2019

Correspondence: Kazuyuki Matsumoto

Department of Gastroenterology and Hepatology, Okayama University Graduate School of Medicine, Dentistry and Pharmaceutical Science, 2-5-1, Shikata-cho, Kita-ku, Okayama 700-8558, Japan

Tel: +81+086-235-7219, Fax: +81+086-225-5991, E-mail: matsumoto.k@okayama-u.ac.jp

ORCID: https://orcid.org/0000-0002-5102-7452

(c) This is an Open Access article distributed under the terms of the Creative Commons Attribution Non-Commercial License (http://creativecommons.org/ licenses/by-nc/3.0) which permits unrestricted non-commercial use, distribution, and reproduction in any medium, provided the original work is properly cited. with poor prognosis, with less than $5 \%$ of patients surviving 5 years after diagnosis. ${ }^{3,4}$ This poor survival is the result of late diagnosis and low rate of complete resection..$^{5-7}$

Surgical resection is the only potential cure for pancreatic cancer, and complete histologic resection with negative margin is an independent predictor of postoperative survival. ${ }^{4}$ In the absence of metastatic lesion, which precludes resection, accurate assessment of vascular invasion, especially the major arteries (superior mesenteric artery [SMA], celiac artery, and common hepatic artery [CHA]) and the major veins (superior mesenteric vein $[\mathrm{SMV}]$ and portal vein $[\mathrm{PV}])$, is an important factor for determining the resectability of pancreatic cancer. ${ }^{8,9}$ Vascular invasion is a relatively frequent discovery in pancreatic cancer, and it is found in $21 \%-64 \%$ of patients, depending on the study population. ${ }^{7,10}$

Although contrast-enhanced computed tomography (CECT) is currently the "gold standard" for preoperative stag- 
ing of pancreatic cancer, not all patients can be evaluated by CECT because of allergy to the contrast agent.

Endoscopic ultrasonography (EUS) is commonly performed to evaluate pancreatic cancer and is considered one of the most accurate preoperative examinations for staging of pancreatic cancer. ${ }^{11,12}$ EUS can accurately evaluate pancreatic cancer without using a contrast agent. EUS has good spatial resolution ability, and some recent studies have revealed the accuracy and interobserver reliability of EUS for diagnosis of vascular invasion and staging of pancreatic cancer. ${ }^{13-16}$ However, there is no consensus on the role of EUS in preoperative imaging assessment of pancreatic cancer.

Our study aimed to examine the diagnostic ability of EUS for vascular invasion in pancreatic cancer, and to compare EUS findings and pathologically measured distances between pancreatic cancers and major vessels.

\section{MATERIALS AND METHODS}

\section{Patients}

The medical records of all patients who underwent EUS for detecting pancreatic cancers before surgical resection at Okayama University Hospital between January 2013 and June 2016 were retrospectively reviewed. All registered patients underwent surgical resection and were confirmed to have pancreatic cancers by histopathological findings obtained from surgical specimens. Patients on neoadjuvant chemotherapy were excluded. All patients had provided written informed consent to undergo EUS. This study was approved by the institutional review board of our hospital.

\section{Procedures of EUS}

EUS examinations were performed by two well-experienced endoscopists using a curved linear array scanning scope (UCT260; Olympus Medical Systems, Tokyo, Japan) with Aloka console (Prosound SSD-a10; HITACHI Aloka, Tokyo, Japan) or UE-ME2 (Olympus Medical Systems) monitor/ processing unit. Fasting patients were examined using EUS under intravenous sedation with midazolam. Locoregional staging was performed according to the general rules for the study of pancreatic cancer by the Japan Pancreas Society. ${ }^{17}$ We divided the EUS findings into four types in accordance with the relationship between tumors and major vessels, namely type 1 , clear invasion, encasement of vessel by a tumor; type 2 , a tumor that contacts a vessel with loss of the hyperechoic vessel layer; type 3, a tumor that contacts a vessel without loss of the hyperechoic vessel layer; and type 4, clear non-invasion, existence of distance between a tumor and a vessel (Fig. 1). We regarded types 1 and type 2 as signs of vascular invasion and types 3 and 4 as signs of vascular non-invasion. We evaluated PV, SMV, and SMA for tumors in the pancreatic head, and splenic vein (SPV) and splenic artery (SPA) for tumors in the pancreatic body or tail. In each case, both the main arteries and veins were evaluated.

\section{Outcome measurements and definitions}

The primary objective of this study was to evaluate the diagnostic accuracy of EUS for vascular invasion in pancreatic cancer. The final diagnosis of vascular invasion was confirmed by pathological evaluation of a surgically resected specimen.

The secondary objective was to compare the EUS finding and pathologically measured distance between pancreatic cancer and the observed vessel. We pathologically measured the distances between the vessels and the pancreatic cancers in cases diagnosed as type 2 and type 3 EUS findings with pathologically vascular non-invasion. We cut the surgically resected specimens into sections with 3-mm intervals and evaluated them pathologically. In this study, we pathologically measured the shortest distance between the pancreatic cancer and the observed vessels, and then used the measured distance. In evaluation of all SMAs, PVs, and SMVs that were not surgically excised together with the tumors, the distances between the pancreatic cancers and resection stumps were regarded as distances between the tumors and vessels. We compared the pathologically measured distances in cases with type 2 EUS
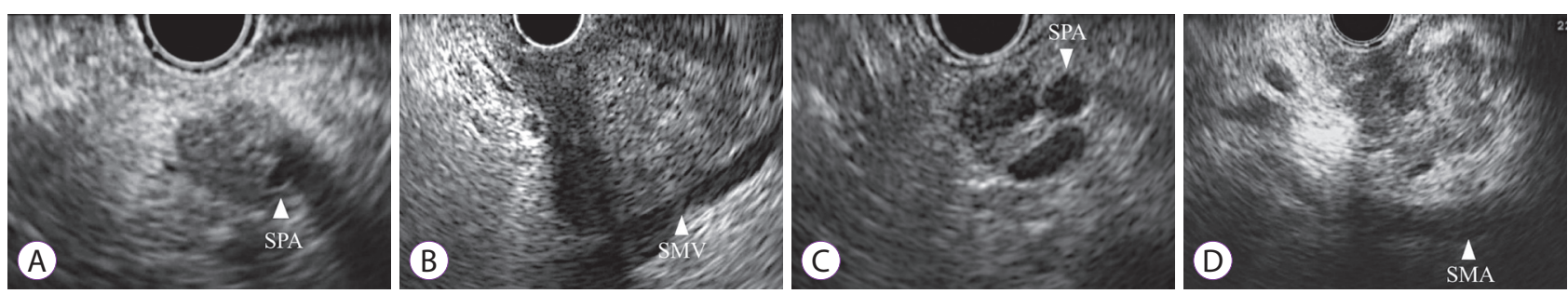

Fig. 1. Classification of endoscopic ultrasonography findings into four types in accordance with the relationship between tumors and major vessels. (A) Type 1: clear invasion, encasement of vessel by tumor. (B) Type 2: a tumor that contacts a vessel with loss of hyperechoic vessel layer. (C) Type 3: a tumor that contacts a vessel without loss of hyperechoic vessel layer. (D) Type 4: clear non-invasion, existence of distance between a tumor and a vessel. SMA, superior mesenteric artery; SMV, superior mesenteric vein; SPA, splenic artery. 
finding and those with type 3 EUS finding.

\section{Statistical analyses}

Categorical variables were reported as percentages and continuous variables, as medians and interquartile range (IQR). The sensitivity, specificity, and accuracy of EUS diagnosis for vascular invasion were calculated with $95 \%$ confidence intervals. Wilcoxon's rank sum test and Kruskal-Wallis test were used to compare continuous data. Fisher's exact test was used to compare categorical data. Statistical analyses were performed using JMP Prol3 for Mac (JMP 13; SAS Institute Inc., Cary, NC, USA).

Table 1. Clinical Characteristics of the Patients

\begin{tabular}{lc}
\hline Parameter & Number \\
\hline Age, median (IQR), yr & $70(65-74)$ \\
Sex, male, $n$ (\%) & $33(58)$ \\
Tumor location, $n(\%)$ & $31(54)$ \\
Head & $18(18)$ \\
Body & $8(14)$ \\
Tail & \\
Preoperative cancer stage & $0 / 3 / 10 / 18 / 25 / 1$ \\
T 0/1a/1b/1c/2/3/4 & $54 / 3$ \\
N 0/1 & $57 / 0$ \\
M 0/1 & $0 / 13 / 18 / 22 / 3 / 1 / 0$ \\
Stage 0/IA/IB/IIA/IIB/III/IV & $26(18-33)$ \\
Tumor size, median (IQR), mm & \\
Histological type & $44(77)$ \\
Well or moderately differentiated tubular & \\
adenocarcinoma, $n$ (\%) & $8(14)$ \\
Poorly differentiated adenocarcinoma, $n(\%)$ & $5(9)$ \\
Others, $n$ (\%) & \\
Measured distance from the resection stump & \\
Major vein, $n$ (\%) & \\
Major artery, $n(\%)$ & \\
\hline Othe & \\
\hline
\end{tabular}

Others, mucinous adenocarcinoma and papillary adenocarcinoma. $\mathrm{IQR}$, interquartile range.

\section{RESULTS}

\section{Patient characteristics}

In total, 57 consecutive patients ( 33 male; median age, 70 [IQR, 65-74] years) were included in this study. Table 1 summarizes the patient characteristics. The preoperative cancer stages of the patients were as follows: 13 patients in Stage IA, 18 patients in Stage IB, 22 patients in Stage IIA, 3 patients in Stage IIB, and 1 patient in Stage III. ${ }^{17}$ The median size of the pancreatic lesions was 26 (IQR, 18-33) mm. Histological types of pancreatic cancers were as follows: well- or moderately differentiated tubular adenocarcinoma in 44 patients (77\%), poorly differentiated adenocarcinoma in 8 patients (14\%), papillary adenocarcinoma in 3 patients (5\%), and mucinous adenocarcinoma in 2 patients (4\%). For measurement of distances between the tumors and vessels, 19 (33\%) cases with a major vein and $34(60 \%)$ cases with a major artery were evaluated to measure the distance from the surgically resected stump. In other cases, the distances between the tumor and surgically resected vessels were measured.

\section{Evaluation of vascular invasion by EUS}

For the 31 tumors located in the pancreatic head, we evaluated 15 SMVs, 16 PVs, and 31 SMAs. Similarly, for the 26 tumors located in the pancreatic body or tail, we evaluated 26 SPVs and 26 SPAs. We evaluated the diagnostic abilities for the major veins (SMV, PV, and SPV) and arteries (SMA and SPA). The sensitivity, specificity, positive predictive value (PPV), negative predictive value (NPV), and accuracy of EUS diagnosis were 89\% (16/18), 92\% (36/39), 84\% (16/19), 95\% $(36 / 38)$, and $91 \%(52 / 57)$, respectively, for the major veins and $83 \%(5 / 6), 94 \%$ (48/51), 63\% (5/8), 98\% (48/49), and 93\% (53/57), respectively, for the major arteries (Table 2).

The relationships between EUS finding and pathological diagnosis for vascular invasion are shown in Tables 3 and 4 . All cases regarded as types 1 and 4 by EUS were correctly diagnosed in both, the major veins and arteries. Three of 14 cases with type 2 and 2 of 23 cases with type 3 were incorrectly diagnosed in the major veins. All 3 cases with type 2 and 1 of 34 cases with type 3 were incorrectly diagnosed in the major arteries.

Table 2. Diagnostic Accuracy of Endoscopic Ultrasonography for Vascular Invasion

\begin{tabular}{lccccc}
\hline & Sensitivity (95\% CI) & $\begin{array}{c}\text { Specificity } \\
(\mathbf{9 5 \%} \text { CI })\end{array}$ & $\begin{array}{c}\text { PPV } \\
(\mathbf{9 5 \%} \text { CI })\end{array}$ & $\begin{array}{c}\text { NPV } \\
(\mathbf{9 5 \%} \text { CI) }\end{array}$ & $\begin{array}{c}\text { Accuracy } \\
(\mathbf{9 5 \%} \text { CI })\end{array}$ \\
\hline Major vein & $89(73-96)$ & $92(85-96)$ & $84(69-91)$ & $95(87-98)$ & $91(81-96)$ \\
Major artery & $83(49-97)$ & $94(90-96)$ & $63(37-73)$ & $98(94-98)$ & $93(86-96)$ \\
\hline
\end{tabular}

$\mathrm{CI}$, confidence interval; NPV, negative predictive value; PPV, positive predictive value. 
Relationship between EUS finding and pathologically evaluated distance between the tumor and vessel

To evaluate the pathological distance between the tumor and major veins, we compared 3 cases with type 2 and 21 cases with type 3 . The pathological distances of 3 cases with type 2 EUS findings were $0 \mu \mathrm{m}, 96 \mu \mathrm{m}$, and $742 \mu \mathrm{m}$. The median distance of 21 cases with type 3 EUS findings was 2833 (range, $508-18,000) \mu \mathrm{m}$. The distances of the cases with type 2 EUS findings were significantly shorter than those of cases with type 3 EUS findings (median [IQR], 96 [0-742] $\mu \mathrm{m}$ vs. 2833 $[1,076-5,694] \mu \mathrm{m}, p=0.012$ ) (Fig. 2A).

To evaluate the pathological distance between a tumor and a major artery, we compared 3 cases with type 2 and 33 cases with type 3 . The distances of 3 cases with type 2 were $0 \mu \mathrm{m}$, $623 \mu \mathrm{m}$, and $854 \mu \mathrm{m}$. The median distance of 33 cases with type 3 was 3,097 (range, 700-18,000) $\mu \mathrm{m}$. The distances of cases with type 2 were also significantly shorter than those of cases with type 3 (median [IQR], 623 [0-854] $\mu \mathrm{m}$ vs. 3,097 $[1,396-6,000] \mu \mathrm{m}, p=0.0061$ ) (Fig. 2B).

Table 3. Relationship between EUS Finding and Pathological Diagnosis for Vascular Invasion in Evaluated Major Vein ( $n=57)$

\begin{tabular}{lcc}
\hline \multirow{2}{*}{ EUS finding } & \multicolumn{2}{c}{ Pathological diagnosis for vascular invasion } \\
\cline { 2 - 3 } & \multicolumn{1}{c}{ Positive, $\boldsymbol{n}(\%)$} & Negative, $\boldsymbol{n}(\%)$ \\
\hline Type 1: Clear invasion $(n=5)$ & $5(100)$ & $0(0)$ \\
Type 2: Loss of extra-hyperechoic vessel layer $(n=14)$ & $11(79)$ & $3(21)$ \\
Type 3: Existence of extra-hyperechoic vessel layer $(n=23)$ & $2(9)$ & $21(91)$ \\
Type 4: Clear non-invasion $(n=15)$ & $0(0)$ & $15(100)$ \\
\hline
\end{tabular}

EUS, endoscopic ultrasonography.

Table 4. Relationship between EUS Finding and Pathological Diagnosis for Vascular Invasion in Evaluated Major Artery ( $n=57)$

\begin{tabular}{lcc}
\hline \multirow{2}{*}{ EUS finding } & \multicolumn{2}{c}{ Pathological diagnosis for vascular invasion } \\
\cline { 2 - 3 } & Positive, $\boldsymbol{n}(\%)$ & Negative, $\boldsymbol{n}(\%)$ \\
\hline Type 1: Clear invasion $(n=5)$ & $5(100)$ & $0(0)$ \\
Type 2: Loss of extra-hyperechoic vessel layer $(n=3)$ & $0(0)$ & $3(100)$ \\
Type 3: Existence of extra-hyperechoic vessel layer $(n=34)$ & $1(3)$ & $33(97)$ \\
Type 4: Clear non-invasion $(n=15)$ & $0(0)$ & $15(100)$ \\
\hline
\end{tabular}

EUS, endoscopic ultrasonography.

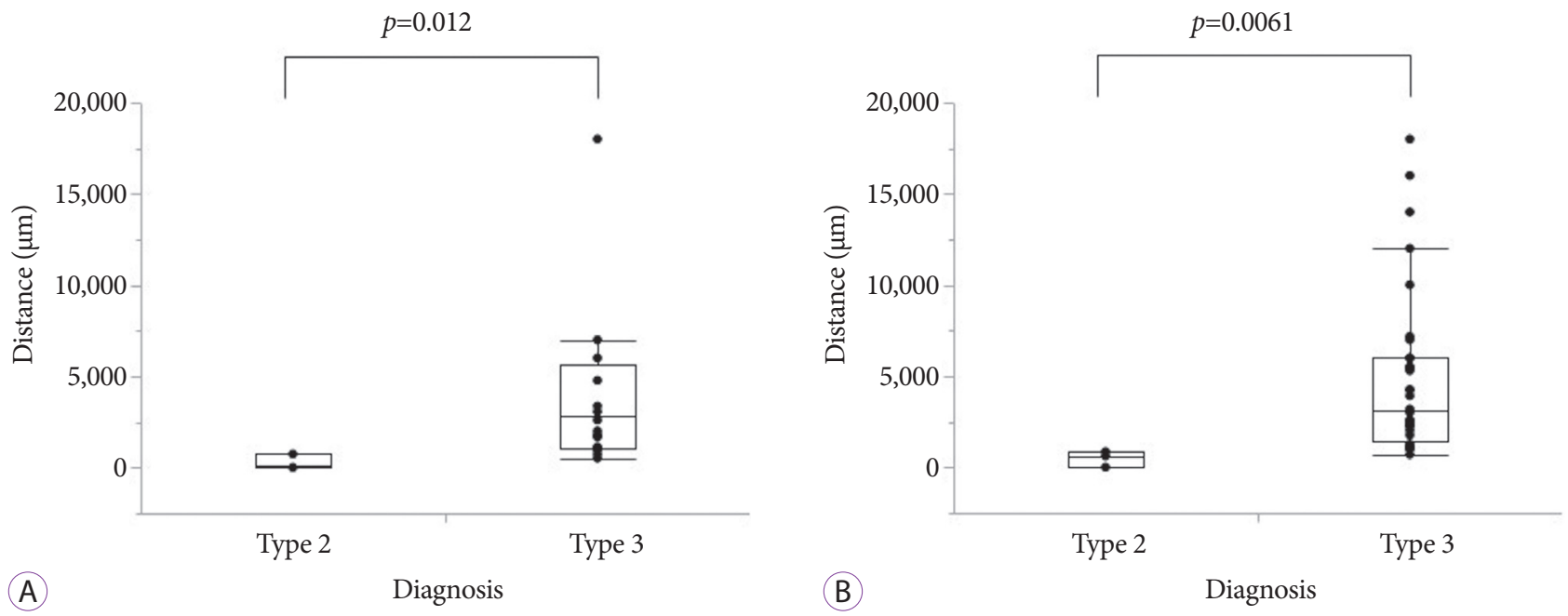

Fig. 2. Box plots of distances between pancreatic cancers and pathologically evaluated vessels. Box plots of the distances from the evaluated major veins (A) and major arteries (B) to pancreatic cancers in cases of type 2 and type 3 endoscopic ultrasonography (EUS) findings with no vascular invasion. The distances of the cases with type 2 EUS finding were significantly shorter than those of cases with type 3 EUS finding in both, the veins and arteries. 
Cases with incurred diagnosis are shown in Table 5. There was little $(<900 \mu \mathrm{m})$ or no distance between the tumors and observed vessels in all false-positive cases (Figs. 3, 4). All tumors with a distance of $\geq 964 \mu \mathrm{m}$ from the major veins and that of $\geq 1,000 \mu \mathrm{m}$ from the major arteries were correctly diagnosed as type 3 .

\section{DISCUSSION}

Accurate evaluation of vascular invasion in patients with pancreatic cancer is very important to determine the resectability of pancreatic cancer and to predict the prognosis. ${ }^{18-23}$ Although CECT is currently the "gold standard" for preoperative staging of pancreatic cancer, not all patients can be

Table 5. Cases with Incorrect Diagnosis by EUS

\begin{tabular}{lccccccc}
\hline Patient No. & EUS finding & $\begin{array}{c}\text { Evaluated } \\
\text { vessel }\end{array}$ & $\begin{array}{c}\text { Vascular } \\
\text { invasion }\end{array}$ & $\begin{array}{c}\text { Distance between } \\
\text { tumor and vessel }(\boldsymbol{\mu m})\end{array}$ & $\begin{array}{c}\text { Tumor loca- } \\
\text { tion }\end{array}$ & $\begin{array}{c}\text { Tumor size } \\
(\mathbf{m m})\end{array}$ & $\begin{array}{c}\text { Histological } \\
\text { type }\end{array}$ \\
\hline 1 & Type 2 & SMV & $(-)$ & 742 & $\mathrm{Ph}($ neck $)$ & 55 & por \\
\hline 2 & Type 2 & SMV & $(-)$ & 96 & $\mathrm{Ph}($ neck) & 33 & tub2 \\
\hline 3 & Type 2 & SMV & $(-)$ & $0^{\text {a) }}$ & $\mathrm{Pt}$ & 65 & muc \\
\hline 4 & Type 2 & SPA & $(-)$ & 623 & $\mathrm{~Pb}$ & 30 & tub2 \\
\hline 5 & Type 2 & SPA & $(-)$ & $0^{\text {a) }}$ & $\mathrm{Pt}$ & 45 & tub2 \\
\hline 6 & Type 2 & SMA & $(-)$ & 854 & $\mathrm{Ph}($ neck) & 25 & tub2 \\
\hline 7 & Type 3 & PV & $(+)$ & - & $\mathrm{Ph}$ & 26 & tub2 \\
\hline 8 & Type 3 & PV & $(+)$ & - & $\mathrm{Ph}$ & 45 & tub1 \\
\hline 9 & Type 3 & SPA & $(+)$ & - & $\mathrm{Pt}$ & 65 & muc \\
\hline
\end{tabular}

Type 2: Loss of the extra-hyperechoic layer (positive invasion).

Type 3: Existence of the extra-hyperechoic layer of the vessel (negative invasion).

EUS, endoscopic ultrasonography; muc, mucinous adenocarcinoma; por, poorly differentiated adenocarcinoma; PV, portal vein; SMA, superior mesenteric artery; SMV, superior mesenteric vein; SPA, splenic artery; tub, tubular adenocarcinoma.

a) Although the tumor cells were neighboring to the vascular walls, no invasion was detected with Elastica Van Gieson staining.
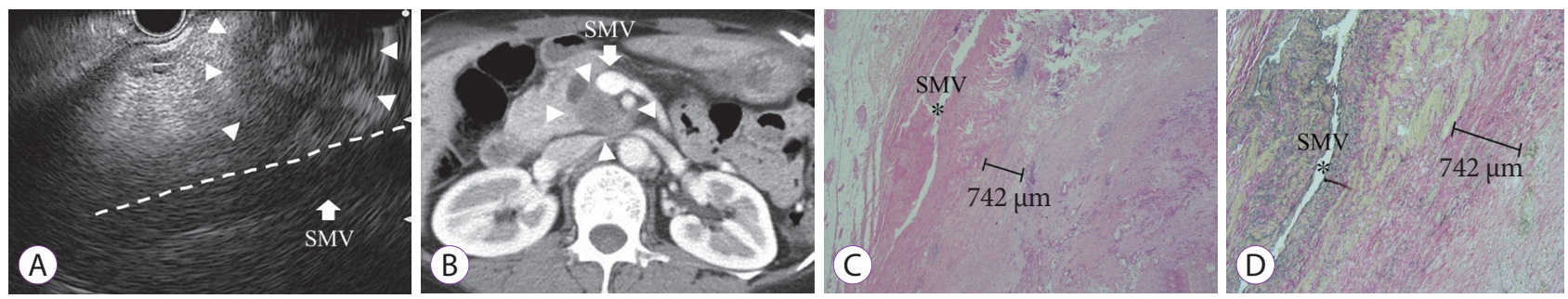

Fig. 3. False-positive case of pancreatic cancer in transition of head and body, with a size of $55 \mathrm{~mm}$. (A) This case was diagnosed as type 2 . The pancreatic tumor (arrowheads) contacting the superior mesenteric vein (SMV: arrow) with the loss of the hyperechoic vessel layer, as observed by endoscopic ultrasonography. (B) Computed tomography image showing pancreatic tumor (arrowheads) contact SMV (arrow). (C) Pathological evaluation showing a distance of $742 \mu \mathrm{m}$ between the tumor and SMV (hematoxylin-eosin stain, $\times 20$ ). (D) No invasion to vessel was verified using Elastica van Gieson staining $(\times 40)$.
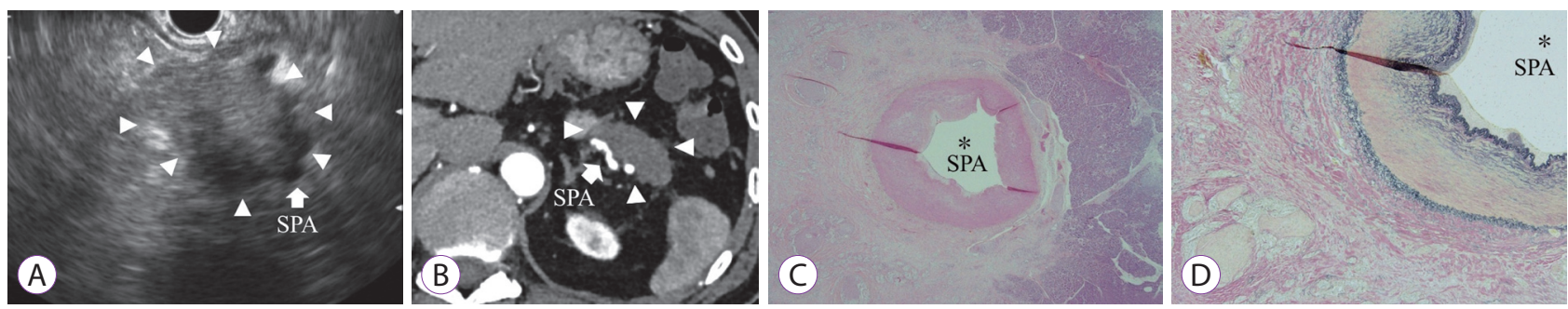

Fig. 4. False-positive case of a pancreatic cancer in tail with a size of $45 \mathrm{~mm}$. (A) This case was diagnosed as type 2. The pancreatic tumor (arrowheads) contacting the splenic artery (SPA: arrow), with the loss of the hyperechoic vessel layer, as observed by endoscopic ultrasonography. (B) Computed tomography image showing the pancreatic tumor (arrowheads) in contact with the SPA (arrow). (C) Pathological evaluation showing a distance of $0 \mu \mathrm{m}$ between the tumor and SPA (hematoxylin-eosin staining, $\times 20$ ). (D) Although proliferation of the tumor cells surrounding the SPA was observed, no invasion to vessel was verified in Elastica van Gieson staining $(\times 40)$. 
evaluated by CECT, because a few patients have allergy to the contrast agent, and some patients have decreased renal function. Approximately $0.6 \%$ of patients experience allergic reactions on administration of iodinated contrast medium. ${ }^{24}$ EUS can accurately evaluate pancreatic cancer without using contrast agent.

Although EUS has recently shown a good ability to detect vascular invasion, evaluation by EUS is highly operator dependent. In a meta-analysis involving evaluation of the vascular invasion ability of EUS and CT in patients with pancreatic cancer, the sensitivity, specificity, PPV, and NPV of EUS were $86 \%, 93 \%, 88 \%$, and $90 \%$, and those of CT were $58 \%, 95 \%$, 90\%, and 75\%. ${ }^{14}$ Although EUS has a higher sensitivity than that of CT, the specificity of both EUS and CT is comparable. In patients who underwent CECT, the sensitivity, specificity, PPV, NPV, and accuracy of CECT were 77\% (13/17), 90\% (36/40), 59\% (13/17), 90\% (36/40), and 86\% (49/57), respectively, for the major veins and 86\% (6/7), 88\% (44/50), 50\% (6/12), $98 \%$ (44/45), and 91\% (52/57), respectively, for the major arteries. Five patients whose major veins were evaluated and four patients whose major arteries were evaluated were correctly diagnosed by EUS; however, the CECT diagnosis was incorrect. For arterial evaluation, the sensitivity of CECT was superior to that of EUS. Conversely, for venous evaluation, the diagnostic ability of EUS was superior to that of CECT. This study suggested that EUS is more accurate than CECT for detection of major vein invasion, which was consistent with previous reports. ${ }^{25,26}$ Zhang et al. reported a meta-analysis that compared CECT and magnetic resonance imaging (MRI) for preoperative vascular evaluation of pancreatic cancers. ${ }^{27}$ The sensitivity and specificity were $71 \%(64 \%-78 \%)$ and $92 \%$ (89\%-95\%), respectively, by CECT, and 67\% (59\%-74\%) and 94\% (91\%-96\%), respectively, by MRI, with no significant difference. Compared to CECT and MRI, magnetic resonance angiography did not provide any additional information on vascular staging in this study.

Our results agree with those of previous studies and indicate that EUS is a good method to evaluate the locoregional staging of pancreatic cancer, especially vascular invasion. ${ }^{13-16}$ The sensitivity, specificity, and accuracy of EUS in this study appeared to be higher than those in several previous studies. However, the sensitivity, specificity, and accuracy of EUS in this study were almost similar to those in recent studies. ${ }^{14,28}$ This similarity could be attributed to the continually improving EUS technique; use of Aloka 10 or UE-ME2 as the monitor/processing unit, yielding a high-resolution EUS image; and performance of EUS examinations by well-experienced endoscopists.

In this study, eight patients were diagnosed with vascular invasion to a major artery. Of these, seven patients were diag- nosed with tumor invasion to the SPA; these patients underwent upfront surgery. One patient with type 2 EUS finding was suspected for invasion to the SMA. The CECT image did not show suspicion of SMA invasion. Therefore, the patient was scheduled for surgery after a discussion among the surgeons. Finally, the evaluation of the surgically resected specimen did not show vascular invasion.

To the best of our knowledge, there is no previous study that pathologically measured the distance between the tumor and the observed vessel, and compared this distance with EUS finding. According to the results of our study, tumors with loss of the extra-hyperechoic vessel layers were significantly nearer from the observed vessels in pathological specimen than those with existence of hyperechoic vessel layers were. There was little $(<900 \mu \mathrm{m})$ or no distance between tumors and vessels in all false-positive cases. All tumors with a distance $\geq 964 \mu \mathrm{m}$ from the major veins and $\geq 1,000 \mu \mathrm{m}$ from the major arteries were correctly diagnosed by EUS.

This study showed that EUS has a high diagnostic ability for vascular invasion. Our result could be useful not only for pancreatic cancer but also for some tumors that may potentially invade a major artery. For example, vascular invasion to the right hepatic artery should be evaluated in patients with bile duct or gallbladder cancer.

This study has some limitations. First, it was retrospectively conducted at a single center, with a relatively small sample size. Second, all EUS examinations were performed with knowledge of prior CT or MRI findings. Third, we evaluated PV, SMV, SPV, SMA, and SPA; however, no case included $\mathrm{CHA}$ as the evaluated vessel in this study. Fourth, in pathological evaluation, we could not directly measure the distance between the tumor and the observed vessel, such as SMA, SMV, and $\mathrm{PV}$, because the vessels were peeled from the tumors in the absence of invasion. Therefore, we regarded the shortest distance between the tumor and cut-off stump as the distance between the tumor and vessel in such cases. Hence, we may have possibly underestimated the distance between the tumor and vessel in this study. Moreover, the distance differed with the slice of the resected specimens and the cutting method used for sectioning the surgically resected specimens.

In conclusion, EUS is a highly useful tool to detect vascular invasion in patients with pancreatic cancer. Our results revealed that the EUS finding of loss of the hyperechoic vessel layers indicated pathologically short distance between the tumor and vessel. Pancreatic cancers with a distance $\geq 1,000 \mu \mathrm{m}$ from the major vessels were correctly diagnosed as vascular non-invasion by EUS.

Conflicts of Interest

The authors have no financial conflicts of interest. 


\section{REFERENCES}

1. Pancreatric Section, British Society of Gastroenterology; Pancreatic Society of Great Britain and Ireland; Association of Upper Gastrointestinal Surgeons of Great Britain and Ireland; Royal College of Pathologists; Special Interest Group for Gastro-Intestinal Radiology. Guidelines for the management of patients with pancreatic cancer periampullary and ampullary carcinomas. Gut 2005;54 Suppl 5:v1-v16.

2. Warshaw AL, Fernández-del Castillo C. Pancreatic carcinoma. N Engl J Med 1992;326:455-465.

3. Jemal A, Siegel R, Xu J, Ward E. Cancer statistics, 2010. CA Cancer J Clin 2010;60:277-300.

4. Wagner M, Redaelli C, Lietz M, Seiler CA, Friess H, Buchler MW. Curative resection is the single most important factor determining outcome in patients with pancreatic adenocarcinoma. Br J Surg 2004;91:586-594.

5. Ahmad NA, Kochman ML, Lewis JD, et al. Endosonography is superior to angiography in the preoperative assessment of vascular involvement among patients with pancreatic carcinoma. J Clin Gastroenterol 2001;32:54-58.

6. DeWitt J, Devereaux B, Chriswell M, et al. Comparison of endoscopic ultrasonography and multidetector computed tomography for detecting and staging pancreatic cancer. Ann Intern Med 2004;141:753-763.

7. Arslan A, Buanes T, Geitung JT. Pancreatic carcinoma: MR, MR angiography and dynamic helical CT in the evaluation of vascular invasion. Eur J Radiol 2001;38:151-159.

8. DiMagno EP, Reber HA, Tempero MA. AGA technical review on the epidemiology, diagnosis, and treatment of pancreatic ductal adenocarcinoma. American Gastroenterological Association. Gastroenterology 1999;117:1464-1484.

9. Rösch T, Dittler HJ, Lorenz R, et al. [The endosonographic staging of pancreatic carcinoma]. Dtsch Med Wochenschr 1992;117:563-569.

10. Megibow AJ, Zhou XH, Rotterdam H, et al. Pancreatic adenocarcinoma: CT versus MR imaging in the evaluation of resectability--report of the Radiology Diagnostic Oncology Group. Radiology 1995;195:327-332.

11. Saftoiu A, Vilmann P. Role of endoscopic ultrasound in the diagnosis and staging of pancreatic cancer. J Clin Ultrasound 2009;37:1-17.

12. Michl P, Pauls S, Gress TM. Evidence-based diagnosis and staging of pancreatic cancer. Best Pract Res Clin Gastroenterol 2006;20:227-251.

13. Dewitt J, Devereaux BM, Lehman GA, Sherman S, Imperiale TF. Comparison of endoscopic ultrasound and computed tomography for the preoperative evaluation of pancreatic cancer: a systematic review. Clin Gastroenterol Hepatol 2006;4:717-725; quiz 664.

14. Nawaz H, Fan CY, Kloke J, et al. Performance characteristics of endoscopic ultrasound in the staging of pancreatic cancer: a meta-analysis. JOP 2013;14:484-497.
15. Yang R, Lu M, Qian X, et al. Diagnostic accuracy of EUS and CT of vascular invasion in pancreatic cancer: a systematic review. J Cancer Res Clin Oncol 2014;140:2077-2086

16. Puli SR, Singh S, Hagedorn CH, Reddy J, Olyaee M. Diagnostic accuracy of EUS for vascular invasion in pancreatic and periampullary cancers: a meta-analysis and systematic review. Gastrointest Endosc 2007;65:788-797.

17. Japan Pancreas Society. Classification of pancreatic carcinoma. 4th ed. Tokyo: Kanehara \& Co., Ltd.; 2017.

18. Nakata B, Nishino H, Ogawa Y, et al. Prognostic predictive value of endoscopic ultrasound findings for invasive ductal carcinomas of pancreatic head. Pancreas 2005;30:200-205.

19. Yeo CJ, Cameron JL, Lillemoe KD, et al. Pancreaticoduodenectomy for cancer of the head of the pancreas. 201 patients. Ann Surg 1995;221:721731; discussion 731-733.

20. Tylén U, Arnesjö B. Resectability and prognosis of carcinoma of the pancreas evaluated by angiography. Scand J Gastroenterol 1973;8:691697.

21. Geer RJ, Brennan MF. Prognostic indicators for survival after resection of pancreatic adenocarcinoma. Am J Surg 1993;165:68-72; discussion $72-73$.

22. Cameron JL, Crist DW, Sitzmann JV, et al. Factors influencing survival after pancreaticoduodenectomy for pancreatic cancer. Am J Surg 1991;161:120-124; discussion 124-125.

23. Launois B, Franci J, Bardaxoglou E, et al. Total pancreatectomy for ductal adenocarcinoma of the pancreas with special reference to resection of the portal vein and multicentric cancer. World J Surg 1993;17:122126; discussion 126-127.

24. ACR Committee on Drugs and Contrast Media. ACR manual on contrast media version 10.3. [Internet]. Washington, D.C.: American College of Radiology; c2017 [updated 2017; cited 2019 Jan 3]. Available from: https://www.acr.org/Quality-Safety/Resources/Contrast-Manual.

25. Buchs NC, Chilcott M, Poletti PA, Buhler LH, Morel P. Vascular invasion in pancreatic cancer: imaging modalities, preoperative diagnosis and surgical management. World J Gastroenterol 2010;16:818-831.

26. Buchs NC, Frossard JL, Rosset A, et al. Vascular invasion in pancreatic cancer: evaluation of endoscopic ultrasonography, computed tomography, ultrasonography, and angiography. Swiss Med Wkly 2007;137:286291.

27. Zhang Y, Huang J, Chen M, Jiao LR. Preoperative vascular evaluation with computed tomography and magnetic resonance imaging for pancreatic cancer: a meta-analysis. Pancreatology 2012;12:227-233.

28. Li JH, He R, Li YM, Cao G, Ma QY, Yang WB. Endoscopic ultrasonography for tumor node staging and vascular invasion in pancreatic cancer: a meta-analysis. Dig Surg 2014;31:297-305. 